\title{
Qualidade de vida de médicos residentes de um hospital escola
}

\author{
Quality of life of medical residents from a teaching hospital
}

\author{
Bruna Arrais Dias ${ }^{1}$, Marília Neves Pereira ${ }^{1}$, Ivone Félix de Sousa ${ }^{2}$, Rogério José de Almeida ${ }^{3} \bowtie$ \\ ${ }^{1}$ Departamento de Medicina da Pontifícia Universidade Católica de Goiás (PUC Goiás). Goiânia, GO. \\ ${ }^{2}$ Departamento de Psicologia da PUC Goiás. Goiânia, GO. \\ ${ }^{3}$ Departamento de Medicina e Programa de Pós-Graduação em Ciências Ambientais e Saúde da PUC Goiás. Goiânia, GO.
}

\section{RESUMO}

Objetivos: Avaliar a qualidade de vida de médicos residentes de um hospital escola situado na cidade de Goiânia, Goiás.

Métodos: Trata-se de um estudo transversal descritivo com abordagem quantitativa. A pesquisa foi realizada por meio de um censo entrevistando todos os médicos residentes do hospital. Foram aplicados dois questionários, sendo um com dados sociodemográficos e ocupacionais e o outro o instrumento World Health Organization Quality of Life - Bref (WHOQOL-Bref), que avalia a qualidade de vida. A análise estatística utilizada foi a paramétrica, com o uso da escala intervalar, análise de variância Scheffé e teste do qui-quadrado, considerando o nível de p $<0,05$.

Resultados: Foram entrevistados 84 médicos residentes, com média de idade de 29,8 $\pm 3,6$ anos, sendo 54 (64,3\%) do sexo feminino e 53 $(63,1 \%)$ solteiros. Trinta e oito entrevistados estavam cumprindo o primeiro ano de residência médica, 39 o segundo ano e sete o terceiro ano.

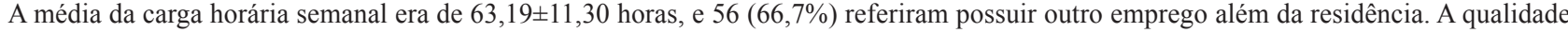
de vida total apresentou o escore $3,44 \pm 0,54$, o domínio físico $3,51 \pm 0,61$, o psicológico $3,43 \pm 0,63$, o de relações sociais $3,42 \pm 0,80$ e o de meio ambiente 3,39 $\pm 0,54$. A percepção da qualidade de vida apresentou um escore de $2,99 \pm 1,00$ e a satisfação pessoal com a saúde de $3,04 \pm 1,17$. Os homens apresentaram escore mais alto para a qualidade de vida total (média 3,64) do que as mulheres (média 3,32) $(p=0,005)$. A diferença significativa entre os sexos apareceu também no domínio físico $(\mathrm{p}=0,005)$ e psicológico $(\mathrm{p}=0,004)$, nos quais as mulheres apresentaram escores mais baixos (médias respectivamente de 3,8 e 3,7) do que os homens (médias respectivamente de 4,3 e 4,2).

Conclusões: A análise geral da qualidade de vida apresentou uma média boa, sendo todos os domínios enquadrados nesta classificação. A autoavaliação evidenciou uma percepção regular da própria qualidade de vida. O sexo feminino foi preditor de pior qualidade de vida geral e nos domínios físico e psicológico.

DESCRITORES: internato e residência; residência médica; qualidade de vida; saúde mental.

\section{ABSTRACT}

Aims: To assess the quality of life of medical residents from a teaching hospital located in Goiânia, State of Goiás, Brazil.

Methods: This is a descriptive cross-sectional study with a quantitative approach. The survey was conducted using a census, during which all medical residents were interviewed. Two questionnaires were applied: one containing sociodemographic and occupational data and the World Health Organization Quality of Life - Bref (WHOQOL-Bref), which assesses quality of life. A parametric statistical analysis was used, including interval scale, Scheffé analysis of variance, and chi-square test. A $\mathrm{p}<0.05$ was considered to be statistically significant.

Results: A total of 84 medical residents with a mean age of $29.8 \pm 3.6$ years were interviewed. Fifty-four $(64.3 \%)$ were female and $53(63.1 \%)$ were single. Thirty-eight were attending the first year, 39 were in the second year, and seven were in the third year of residency. The weekly workload showed an average of $63.19 \pm 11.30$ hours and $56(66.7 \%)$ reported having a job besides the residency program. A score of $3.44 \pm 0.54$ was obtained for the overall quality of life, $3.51 \pm 0.61$ for the physical domain, $3.43 \pm 0.63$ for the psychological domain, $3.42 \pm 0.80$ for social relations, and 3.39 \pm 0.54 for environment. The perception about quality of life showed a score of $2.99 \pm 1.00$ whereas that of personal satisfaction with health was equal to $3.04 \pm 1.17$. Men had a higher score for overall quality of life (mean of 3.64) than women (mean of 3.32) ( $\mathrm{p}=0.005)$. A significant difference between male and female residents was also observed in the physical domain $(\mathrm{p}=0.005)$ and in the psychological one $(\mathrm{p}=0.004)$, in which women had lower scores (means of 3.8 and 3.7, respectively) than men (means of 4.3 and 4.2 , respectively).

Conclusions: The overall quality of life assessment yielded a good mean, and all domains were included under this classification. Selfassessment demonstrated regular perception about the residents' own quality of life. Being a female was a predictor of worse overall quality of life and worse scores in the physical and psychological domains.

KEY WORDS: internship and residency; residency, medical; quality of life; mental health.

Recebido: novembro, 2015

Aceito: março, 2016

Este artigo está licenciado sob forma de uma licença Creative Common 
Abreviaturas: WHOQOL-Bref, World Health Organization Quality of Life-Bref; ANOVA, análise de variância.

\section{INTRODUÇÃO}

A qualidade de vida é entendida como sendo a percepção do indivíduo sobre sua vida, como parte de um contexto cultural cujos valores são vividos e em relação aos quais seus objetivos, expectativas, padrões e preocupações são representados [1]. É uma compreensão do indivíduo de forma holística, englobando a sua saúde física, o estado psicológico, o nível de independência, as interações sociais, as crenças pessoais e a relação com o meio ambiente [2].

Diversos são os fatores envolvidos no bem-estar das pessoas que podem influenciar direta ou indiretamente o seu desenvolvimento pessoal e o desempenho de suas atividades. $O$ indivíduo com prejuízo na qualidade de vida tem uma tendência a sentir-se mais cansado, exigido física e emocionalmente, sem o equilíbrio necessário para manter uma boa saúde e, consequentemente, podendo vir a adoecer [3].

A formação do médico é complexa, desenvolvendose ao longo de vários anos de estudo de graduação e, geralmente, consolidando-se na residência médica [4]. Esta, embora receba várias críticas quanto à oferta de supervisão e orientação qualificadas e aos malefícios de uma extensa carga horária e privação do sono, ainda tem sido reconhecida como o melhor sistema de capacitação profissional voltada para o aperfeiçoamento do médico [4,5].

A residência médica é considerada um momento de formação de intenso estresse, em que o médico está sob constante pressão [6]. O residente é exposto cotidianamente a ambientes e situações carregados de muita exigência e competição, que podem desencadear alterações de comportamento não desejáveis e quadros de depressão e ansiedade [7,8]. Por outro lado, há quem considere o estresse como um elemento normal, que pode até promover efeitos desejáveis, tais como tolerância, autoconfiança e maturidade, e ainda estimular a busca constante por conhecimento e habilidades inerentes ao profissional médico [9].

É fato já demonstrado na literatura que os médicos residentes compõem um grupo de risco para distúrbios emocionais e comportamentais [4-8,10,11]. Elevado grau de sofrimento emocional, suicídio e abuso de álcool e de outras drogas também têm sido observados especificamente nessa população [12].

O foco deste estudo situa-se na questão da qualidade de vida de médicos residentes. O estudo teve como objetivo avaliar a qualidade de vida dos médicos residentes de um Hospital Escola da cidade de Goiânia, capital do estado de Goiás.

\section{MÉTODOS}

Trata-se de um estudo transversal descritivo com abordagem quantitativa [13]. A pesquisa foi realizada por meio de um censo, ou seja, foram entrevistados todos os médicos residentes que compunham o quadro de residentes regularmente matriculados em um Hospital Escola da cidade de Goiânia/GO, no período da coleta de dados, que ocorreu entre os meses de maio a julho de 2015.

Neste censo, foram entrevistados médicos residentes nas diferentes especialidades médicas, divididas em clínicas e cirúrgicas. As especialidades clínicas abarcaram a Cardiologia, Clínica Médica, Medicina de Família e Comunidade, Medicina Intensiva, Nefrologia, Neurologia Clínica e Pediatria. E as especialidades cirúrgicas abarcaram a Anestesiologia, Cirurgia do Aparelho Digestivo, Cirurgia Geral, Cirurgia Plástica, Cirurgia Torácica, Cirurgia Vascular, Ginecologia e Obstetrícia e Urologia.

Para o desenvolvimento da pesquisa foram utilizados dois instrumentos, sendo o primeiro um questionário semiestruturado para avaliar as questões sociodemográficas ocupacionais. O questionário continha perguntas referentes a especialidades médicas, período de residência, idade, sexo, estado civil, carga horária de trabalho semanal, se possuía outro emprego e se existia apoio psicológico ou psicopedagógico no hospital para os residentes.

O segundo instrumento utilizado foi o questionário para avaliar a qualidade de vida World Health Organization Quality of Life-Bref (WHOQOL-Bref). Esse questionário, desenvolvido pela Organização Mundial de Saúde, foi adaptado e validado no Brasil por Fleck et al. [14]. O instrumento busca avaliar a qualidade de vida dos indivíduos dentro de uma perspectiva transcultural e internacional [15].

No WHOQOL-Bref, as respostas seguem uma escala Likert que varia de 1 a 5 , ou seja, quanto maior for a pontuação (escore) melhor a qualidade de vida. Esse questionário é constituído por 26 questões. Destas, duas são para a autoavaliação (uma avalia percepção da qualidade de vida e a outra a satisfação com a saúde). As demais 24 questões são subdivididas em quatro domínios: físico, psicológico, relações sociais e meio ambiente. Os domínios são representados por facetas e cada faceta é composta por uma pergunta [14]. 
O WHOQOL-Bref foi avaliado a partir de uma sintaxe proposta pelo "WHOQOL Group" [16,17]. Por meio do SPSS 18, primeiramente foram invertidos os valores da escala para as facetas 3, 4 e 26; em seguida, agruparam-se as facetas em domínios; depois foram calculados os escores de cada domínio (de 1 a 5 , por meio da média) e finalmente, os escores foram transformados em escalas para cada domínio ( 0 a 100 , por meio da fórmula [(Média -4$) \times(100 / 16)])$. Para analisar a qualidade de vida por meio dos escores do WHOQOL-Bref, leva-se em consideração as categorias de resultados: de 1 a 1,99 necessita melhorar; de 2 a 2,99 regular; de 3 a 3,99 boa; e de 4 a 5 muito boa $[6,16,17]$.

Após a aplicação dos instrumentos, foi confeccionado um banco de dados utilizando o software IBM SPSS Statistics 18. Por meio deste, foi realizada uma análise descritiva das variáveis referentes ao tema em estudo, utilizando-se média, frequência e desvio padrão. Os testes utilizados para avaliar se existiu ou não diferença estatisticamente significativa $(p \leq 0,05)$ entre amostras independentes e múltiplas variáveis, foram o teste $\mathrm{t}$ de Student e o teste de análise de variância (ANOVA) Scheffé, respectivamente.

Todos os itens do questionário sociodemográfico ocupacional foram categorizados e descritos por meio da frequência, porcentagem e mediana, com exceção da idade, que foi tratada como variável escalar e, portanto, foram descritos frequência, porcentagem, média e desvio padrão.

Antes da aplicação do questionário, o médico residente lia e assinava o Termo de Consentimento Livre e Esclarecido, que deixava clara a garantia de sigilo sobre a identidade do participante. A pesquisa foi registrada na Plataforma Brasil do Ministério da Saúde sob protocolo CAAE: 39325514.0.0000.0037, aprovada pelo Comitê de Ética em Pesquisa da Pontifícia Universidade Católica de Goiás com o parecer no 923.616 e pelo Comitê de Ética em Pesquisa do Hospital Santa Casa de Misericórdia de Goiânia com o parecer $\mathrm{n}$ o 927.982 .

\section{RESULTADOS}

A pesquisa incluiu todos os 84 médicos residentes do hospital pesquisado, sem que tenha havido nenhuma exclusão. Entre os 84 médicos, 41 (48,8\%) atuavam em especialidades clínicas e 43 (51,2\%) em especialidades cirúrgicas. Trinta e oito médicos $(45,2 \%)$ cursavam o primeiro ano de residência, $39(46,4 \%)$ cursavam o segundo ano e sete $(8,3 \%)$ cursavam o terceiro ano.

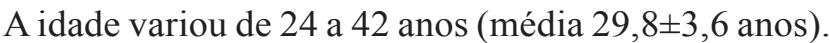

A maioria dos residentes eram do sexo feminino $(64,3 \%)$ e solteiros $(63,1 \%)$ (Tabela 1$)$.

Em relação à carga horária semanal dedicada à residência médica no hospital, houve uma variação entre 40 a 100 horas de dedicação, que foram distribuídas em categorias conforme a Tabela 1 . Houve predominância na categoria de 40 a 60

Tabela 1. Descrição das variáveis sócio-demográficasocupacionais dos 84 médicos residentes do hospital escola.

\begin{tabular}{|c|c|c|}
\hline Variáveis & $\mathrm{n}$ & $\%$ \\
\hline \multicolumn{3}{|l|}{ Especialidades médicas } \\
\hline Clínicas & 41 & 48,8 \\
\hline Cirúrgicas & 43 & 51,2 \\
\hline \multicolumn{3}{|l|}{ Período da residência } \\
\hline R1 (primeiro ano) & 38 & 45,2 \\
\hline R2 (segundo ano) & 39 & 46,4 \\
\hline R3 (terceiro ano) & 7 & 8,3 \\
\hline \multicolumn{3}{|l|}{ Idade (média 29,1 $\pm 3,56$ ) } \\
\hline 24 anos & 3 & 3,6 \\
\hline 25 anos & 9 & 10,7 \\
\hline 26 anos & 9 & 10,7 \\
\hline 27 anos & 14 & 16,7 \\
\hline 28 anos & 7 & 8,3 \\
\hline 29 anos & 7 & 8,3 \\
\hline 30 anos & 10 & 11,9 \\
\hline 31 anos & 5 & 6 \\
\hline 32 anos & 9 & 10,7 \\
\hline 33 anos & 3 & 3,6 \\
\hline 34 anos & 2 & 2,4 \\
\hline 35 anos & 2 & 2,4 \\
\hline 36 anos & 2 & 2,4 \\
\hline 41 anos & 1 & 1,2 \\
\hline 42 anos & 1 & 1,2 \\
\hline \multicolumn{3}{|l|}{ Sexo } \\
\hline Feminino & 54 & 64,3 \\
\hline Masculino & 30 & 35,7 \\
\hline \multicolumn{3}{|l|}{ Estado civil } \\
\hline Solteiro & 53 & 63,1 \\
\hline Casado & 30 & 35,7 \\
\hline Divorciado & 1 & 1,2 \\
\hline \multicolumn{3}{|l|}{ Carga horária de trabalho } \\
\hline de 40 a 60 horas & 66 & 78,6 \\
\hline de 61 a 80 horas & 10 & 11,9 \\
\hline maior do que 80 horas & 8 & 9,5 \\
\hline \multicolumn{3}{|l|}{ Possui outro emprego? } \\
\hline Sim & 56 & 66,7 \\
\hline Não & 28 & 33,3 \\
\hline \multicolumn{3}{|c|}{$\begin{array}{l}\text { A instituição oferece apoio psicológico ou } \\
\text { psicopedagógico? }\end{array}$} \\
\hline Sim & 3 & 3,6 \\
\hline Não & 49 & 58,3 \\
\hline Não sei & 32 & 38,1 \\
\hline
\end{tabular}


horas semanais $(78,6 \%)$. Observou-se que $66,7 \%$ participantes informaram possuir outro emprego além da residência. Em relação à pergunta se a instituição oferecia algum apoio psicológico e/ou psicopedagógico, $58,3 \%$ responderam que não.

A análise geral da qualidade de vida do grupo de residentes, realizada por meio dos dados coletados com

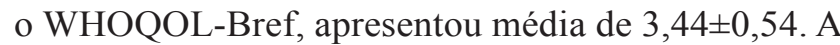
percepção da qualidade de vida apresentou um escore de 2,99 $\pm 1,00$ e a satisfação pessoal com a saúde de 3,04 $\pm 1,17$ (Tabela 2).
Ao analisar cada domínio separadamente, todos os domínios apresentaram escores indicando boa qualidade de vida (físico, psicológico, relações sociais e meio ambiente), variando a média de 3,39 a 3,51 (Tabela 2). No entanto, quando se avaliam as facetas que compõem os domínios, observa-se que algumas delas apresentaram médias que indicam qualidade de vida regular. São elas: no domínio físico, "o sono" (média 2,58), no domínio psicológico, "aproveitar a vida" (média 2,74), e no domínio meio ambiente o "ambiente físico" (média 2,63) e o "lazer" (média 2,43) (Tabela 3).

Tabela 2. Análise da qualidade de vida medida pelo WHOQOL-Bref nos 84 médicos residentes do hospital escola.

\begin{tabular}{|c|c|c|c|c|}
\hline & Médias das escalas & Desvio padrão & Médias dos escores & Desvio padrão \\
\hline Domínio físico & 62,67 & 15,308 & 3,51 & 0,61 \\
\hline Domínio psicológico & 60,71 & 15,860 & 3,43 & 0,63 \\
\hline Domínio relações sociais & 60,52 & 20,045 & 3,42 & 0,80 \\
\hline Domínio meio ambiente & 59,86 & 13,587 & 3,39 & 0,54 \\
\hline Percepção da Qualidade de Vida & 49,7 & 24,998 & 2,99 & 1,00 \\
\hline Satisfação pessoal com a saúde & 50,89 & 29,156 & 3,04 & 1,17 \\
\hline Qualidade de vida total & 60,97 & 13,434 & 3,44 & 0,54 \\
\hline
\end{tabular}

Tabela 3. Domínios e facetas do WHOQOL-Bref nos 84 médicos residentes do hospital escola.

\begin{tabular}{|c|c|c|c|}
\hline Domínio e facetas correspondentes & Mediana & Média & Desvio padrão \\
\hline \multicolumn{4}{|l|}{ FÍSICO } \\
\hline 3. Em que medida você acha que sua dor (física) impede você de fazer o que você precisa? & 4 & 3,89 & 1,12 \\
\hline 4. O quanto você precisa de algum tratamento médico para levar sua vida diária? & 4 & 4,12 & 0,97 \\
\hline 10. Você tem energia suficiente para seu dia-a-dia? & 3 & 3,11 & 0,93 \\
\hline 15. Quão bem você é capaz de se locomover? & 4,5 & 4,43 & 0,65 \\
\hline 16. Quão satisfeito (a) você está com o seu sono? & 2 & 2,58 & 1,04 \\
\hline 17. Quão satisfeito (a) você está com sua capacidade de desempenhar as atividades do seu dia-a-dia? & 3 & 3,06 & 0,90 \\
\hline 18. Quão satisfeito (a) você está com sua capacidade para o trabalho? & 3,5 & 3,36 & 0,82 \\
\hline \multicolumn{4}{|l|}{ PSICOLÓGICO } \\
\hline 5. O quanto você aproveita a vida? & 3 & 2,74 & 0,87 \\
\hline 6. Em que medida você acha que a sua vida tem sentido? & 4 & 4,31 & 0,78 \\
\hline 7. O quanto você consegue se concentrar? & 3 & 3,32 & 0,76 \\
\hline 11. Você é capaz de aceitar sua aparência física? & 4 & 3,55 & 1,06 \\
\hline 19. Quão satisfeito (a) você está consigo mesmo? & 4 & 3,32 & 1,02 \\
\hline 26. Com que frequência você tem sentimentos negativos tais como mau humor, desespero, ansiedade, depressão? & 4 & 3,33 & 1,01 \\
\hline \multicolumn{4}{|l|}{ RELAÇÕES SOCIAIS } \\
\hline 20. Quão satisfeito (a) você está com suas relações pessoais (amigos, parentes, conhecidos, colegas)? & 3 & 3,15 & 1,06 \\
\hline 21. Quão satisfeito (a) você está com sua vida sexual? & 4 & 3,43 & 1,04 \\
\hline 22. Quão satisfeito (a) você está com o apoio que você recebe de seus amigos? & 4 & 3,68 & 0,84 \\
\hline \multicolumn{4}{|l|}{ MEIO AMBIENTE } \\
\hline 8. Quão seguro (a) você se sente em sua vida diária? & 3 & 3,44 & 0,90 \\
\hline 9. Quão saudável é o seu ambiente físico (clima, barulho, poluição, atrativos)? & 3 & 2,63 & 1,03 \\
\hline 12. Você tem dinheiro suficiente para satisfazer suas necessidades? & 3 & 3,00 & 0,99 \\
\hline 13. Quão disponíveis para você estão as informações que precisa no seu dia-a-dia? & 3 & 3,42 & 0,88 \\
\hline 14. Em que medida você tem oportunidades de atividade de lazer? & 2 & 2,43 & 0,72 \\
\hline 23. Quão satisfeito (a) você está com as condições do local onde mora? & 4 & 4,27 & 0,70 \\
\hline 24. Quão satisfeito (a) você está com o seu acesso aos serviços de saúde? & 4 & 3,61 & 1,08 \\
\hline 25. Quão satisfeito (a) você está com o seu meio de transporte? & 4 & 4,36 & 0,71 \\
\hline
\end{tabular}


Ao avaliar a relação entre percepção de qualidade de vida, satisfação com a saúde e qualidade vida total do WHOQOL-Bref com as variáveis sociodemográficas ocupacionais, observou-se que só houve diferença estatisticamente significativa na relação entre sexo e qualidade de vida total, indicando que os homens apresentavam melhor qualidade de vida (média 3,64) do que as mulheres (média 3,32), $(\mathrm{p}=0,005)$ (Tabela 4).

Duas variáveis apresentaram significância limítrofe na relação com a qualidade de vida: maior carga horária de trabalho com pior satisfação com a saúde $(\mathrm{p}=0,052)$; e ter outro emprego com pior percepção da qualidade de vida $(\mathrm{p}=0,064)$ (Tabela 4).

Além do escore geral, a diferença significativa entre os sexos apareceu também no domínio físico $(\mathrm{p}=0,005)$ e psicológico $(\mathrm{p}=0,004)$, nos quais as mulheres apresentaram escores mais baixos (médias respectivamente de 3,8 e 3,7 ) do que os homens (médias respectivamente de 4,3 e 4,2) (Tabela 5).

Tabela 4. Análise dos aspectos da qualidade de vida e do WHOQOL-Bref nos 84 médicos residentes do hospital escola.

\begin{tabular}{|c|c|c|c|c|}
\hline & & & da qualidade de vida & \\
\hline & $\mathbf{n}$ & Percepção da QV & Satisfação com a saúde & WHOQOL-Bref total \\
\hline Sexo & & & & \\
\hline Feminino & 54 & $2,83 \pm 0,986$ & $2,85 \pm 1,089$ & $3,323 \pm 0,543$ \\
\hline Masculino & 30 & $3,27 \pm 0,980$ & $3,37 \pm 1,245$ & $3,647 \pm 0,467$ \\
\hline $\mathrm{p}^{*}$ & & 0,057 & 0,063 & 0,005 \\
\hline Ano de Residência & & & & \\
\hline R1 & 38 & $3,21 \pm 0,935$ & $3,11 \pm 1,158$ & $3,49 \pm 0,554$ \\
\hline R2 & 39 & $2,82 \pm 0,970$ & $2,90 \pm 1,142$ & $3,41 \pm 0,508$ \\
\hline R3 & 7 & $2,71 \pm 1,380$ & $3,43 \pm 1,397$ & $3,34 \pm 0,658$ \\
\hline $\mathrm{p} t$ & & 0,175 & 0,734 & 0,344 \\
\hline Estado Civil & & & & \\
\hline Solteiro & 53 & $2,92 \pm 0,895$ & $3,00 \pm 1,109$ & $3,47 \pm 0,548$ \\
\hline Casado & 30 & $3,13 \pm 1,167$ & $3,13 \pm 1,279$ & $3,41 \pm 0,511$ \\
\hline $\mathrm{p}^{*}$ & & 0,364 & 0,635 & 0,645 \\
\hline Ter outro emprego & & & & \\
\hline Sim & 56 & $2,84 \pm 0,949$ & $3,07 \pm 1,126$ & $3,43 \pm 0,510$ \\
\hline Não & 28 & $3,29 \pm 1,049$ & $2,96 \pm 1,261$ & $3,46 \pm 0,598$ \\
\hline $\mathrm{p}^{*}$ & & 0,064 & 0,706 & 0,775 \\
\hline Carga horária de trabalho & & & & \\
\hline De 40 a 65 horas & 66 & $3,03 \pm 0,976$ & $3,17 \pm 1,145$ & $3,46 \pm 0,513$ \\
\hline De 70 a 80 horas & 10 & $2,90 \pm 1,101$ & $2,90 \pm 1,197$ & $3,48 \pm 0,695$ \\
\hline Maior do que 80 horas & 8 & $2,75 \pm 1,165$ & $2,13 \pm 0,991$ & $3,23 \pm 0,548$ \\
\hline $\mathrm{p}+$ & & 0,728 & 0,052 & 0,510 \\
\hline Especialidades Médicas & & & & \\
\hline Clínicas & 41 & $3,10 \pm 1,044$ & $3,00 \pm 1,118$ & $3,37 \pm 0,579$ \\
\hline Cirúrgicas & 43 & $2,88 \pm 0,956$ & $3,07 \pm 1,223$ & $3,50 \pm 0,492$ \\
\hline$p^{*}$ & & 0,331 & 0,785 & 0,254 \\
\hline
\end{tabular}

* Teste t de Student para amostras independentes.

+ Teste ANOVA Scheffé.

QV, qualidade de vida; R1, primeiro ano da residência médica; R2, segundo ano da residência médica; R3, terceiro ano da residência médica. 
Tabela 5. Escores dos domínios de qualidade de vida do WHOQOL-Bref nos 84 médicos residentes do hospital escola.

\begin{tabular}{|c|c|c|c|c|c|}
\hline & \multicolumn{5}{|c|}{ Domínios WHOQOL-Bref } \\
\hline & $\mathbf{n}$ & $\begin{array}{c}\text { FísICO } \\
\text { (média } \pm \text { DP) }\end{array}$ & $\begin{array}{l}\text { PSICOLÓGICO } \\
\text { (média } \pm \text { DP) }\end{array}$ & $\begin{array}{l}\text { RELAÇÕES SOCIAIS } \\
\text { (média } \pm \text { DP) }\end{array}$ & $\begin{array}{l}\text { MEIO AMBIENTE } \\
(\text { média } \pm \text { DP) }\end{array}$ \\
\hline Geral & 84 & $3,51 \pm 0,61$ & $3,43 \pm 0,63$ & $3,42 \pm 0,80$ & $3,39 \pm 0,54$ \\
\hline \multicolumn{6}{|l|}{ Sexo } \\
\hline Feminino & 54 & $3,8 \pm 0,68$ & $3,7 \pm 0,66$ & $3,7 \pm 0,81$ & $3,7 \pm 0,60$ \\
\hline Masculino & 30 & $4,3 \pm 0,69$ & $4,2 \pm 0,65$ & $3,9 \pm 0,58$ & $3,9 \pm 0,61$ \\
\hline $\mathrm{p}^{*}$ & & 0,005 & 0,004 & 0,213 & 0,197 \\
\hline \multicolumn{6}{|l|}{ Ano de Residência } \\
\hline $\mathrm{R} 1$ & 38 & $4,1 \pm 0,66$ & $4,0 \pm 0,62$ & $3,8 \pm 0,73$ & $3,8 \pm 0,62$ \\
\hline R2 & 39 & $3,9 \pm 0,76$ & $3,8 \pm 0,68$ & $3,8 \pm 0,76$ & $3,7 \pm 0,59$ \\
\hline R3 & 7 & $3,7 \pm 0,76$ & $3,6 \pm 0,98$ & $3,6 \pm 0,79$ & $4 \pm 0,58$ \\
\hline $\mathrm{p}+$ & & 0,213 & 0,084 & 0,831 & 0,394 \\
\hline \multicolumn{6}{|l|}{ Estado Civil } \\
\hline Solteiro & 53 & $4,0 \pm 0,72$ & $4,0 \pm 0,62$ & $3,9 \pm 0,72$ & $3,8 \pm 0,64$ \\
\hline Casado & 30 & $3,9 \pm 0,69$ & $3,8 \pm 0,77$ & $3,7 \pm 0,76$ & $3,7 \pm 0,52$ \\
\hline $\mathrm{p}^{*}$ & & 0,600 & 0,211 & 0,195 & 0,483 \\
\hline \multicolumn{6}{|l|}{ Ter outro emprego } \\
\hline Sim & 56 & $3,9 \pm 0,70$ & $3,9 \pm 0,72$ & $3,8 \pm 0,68$ & $3,8 \pm 0,59$ \\
\hline Não & 28 & $4,0 \pm 0,74$ & $3,9 \pm 0,63$ & $3,8 \pm 0,86$ & $3,8 \pm 0,63$ \\
\hline $\mathrm{p}^{*}$ & & 0,590 & 0,911 & 0,836 & 1,000 \\
\hline \multicolumn{6}{|l|}{ Carga horária de trabalho } \\
\hline De 40 a 65 horas & 66 & $4,0 \pm 0,64$ & $3,9 \pm 0,64$ & $3,8 \pm 0,69$ & $3,8 \pm 0,61$ \\
\hline De 70 a 80 horas & 10 & $4,0 \pm 0,82$ & $3,9 \pm 0,99$ & $3,6 \pm 0,97$ & $3,8 \pm 0,63$ \\
\hline Maior do que 80 horas & 8 & $3,6 \pm 1,06$ & $3,5 \pm 0,53$ & $3,8 \pm 0,89$ & $3,5 \pm 0,53$ \\
\hline $\mathrm{p}+$ & & 0,343 & 0,255 & 0,644 & 0,373 \\
\hline \multicolumn{6}{|l|}{ Especialidades Médicas } \\
\hline Clínicas & 41 & $3,9 \pm 0,66$ & $3,8 \pm 0,71$ & $3,7 \pm 0,85$ & $3,7 \pm 0,63$ \\
\hline Cirúrgicas & 43 & $4 \pm 0,75$ & $4 \pm 0,65$ & $3,9 \pm 0,61$ & $3,8 \pm 0,57$ \\
\hline$p^{*}$ & & 0,356 & 0,322 & 0,171 & 0,426 \\
\hline
\end{tabular}

* Teste t de Student para amostras independentes.

+ Teste ANOVA Scheffé.

DP, desvio padrão; R1, primeiro ano da residência médica; R2, segundo ano da residência médica; R3, terceiro ano da residência médica.

\section{DISCUSSÃO}

O sexo feminino foi identificado no presente estudo como um importante preditor de pior avaliação da qualidade de vida. Uma pesquisa com médicos residentes em Granada, Espanha, identificou escores de qualidade de vida mais baixos nas mulheres do que nos homens. As mulheres apresentavam também uma pior percepção da sua qualidade de vida do que os homens que executavam o mesmo trabalho [18]. O sexo feminino como variável preditora de pior qualidade de vida vem sendo justificado por ser a mulher mais cuidadosa e atenta, e possuir uma visão mais crítica, além de preocupar-se e dedicar-se mais à sua saúde [6].
Estudos apontam que as mulheres possuem maior tendência aos sintomas depressivos e ao esgotamento $[3,12]$, o que pode influenciar negativamente na qualidade de vida. Outra explicação aventada para o encontro de pior qualidade de vida no sexo feminino é de que a mulher apresenta dupla jornada de trabalho, no lar e fora dele, sendo sobrecarregada pelos papéis sociais e familiares que lhe são delegados pela sociedade [7].

Observa-se também uma tendência de as mulheres optarem por uma jornada de trabalho parcial, de trabalharem menos horas semanais, assumirem menor volume de serviços e optarem por uma vida profissional mais curta que os homens. As mulheres também fazem 
menos plantões em serviços e são menos propensas a migrações territoriais [19].

Este resultado do presente estudo é importante quando se leva em conta que nos últimos anos a medicina no Brasil vem passando por uma mudança demográfica, com uma tendência consolidada de feminização da profissão médica [19]. Enfatiza-se esse contexto demográfico relacionado à qualidade de vida, já que o envolvimento das profissionais médicas é que deve garantir o futuro da saúde da população [18].

Quase $70 \%$ dos residentes incluídos neste estudo referiam ter outro emprego além da residência médica. Os resultados apresentaram significância limítrofe em relação à influência negativa da presença de outro emprego na qualidade de vida. Um estudo com residentes em São Paulo também evidenciou que mais da metade dos participantes tinham atividades remuneradas externas à residência médica. Os autores consideraram que possíveis fatores que levam o residente a procurar outro emprego seriam o baixo valor da bolsa de estudos associado às excessivas despesas de morar sozinho [20].

No Brasil, a lei 6.932, que regulamenta a residência médica, estipula uma carga horária máxima de 60 horas semanais, incluindo um máximo de 24 horas de plantão [21]. No presente estudo, a carga horária que predominou foi de acordo com a determinada pela lei, havendo uma pequena parcela que excedeu o valor máximo estipulado. Já na literatura, vários estudos identificaram predomínio de uma carga horária acima da preconizada $[6,20]$.

No presente estudo, a carga horária de trabalho semanal relacionou-se de forma indiretamente proporcional à qualidade de vida, com significância limítrofe, a qual pode ser devida ao insuficiente tamanho amostral. Uma grande carga horária de trabalho proporciona menos tempo para o descanso físico e psíquico, e também para desfrutar do convívio de amigos e familiares. Extensas jornadas de trabalho e pouco tempo para lazer e descanso já foram descritos como possíveis preditores de esgotamento profissional $[20,22]$.

No WHOQOL-Bref, a faceta que avalia o quão satisfeita a pessoa está com o seu sono apresentou-se como regular, o que indica que o tempo de sono dos participantes não é suficiente para obter o descanso. Outros estudos apontam que a quantidade de sono insuficiente e a má qualidade do sono podem promover queda na produtividade, desmotivação, baixo desempenho nos estudos e no raciocínio, prejuízo nas relações sociais, dor crônica e um maior risco de acidentes no trabalho e no trânsito $[23,24]$.
A sobrecarga física e emocional durante a assistência aos pacientes é muitas vezes negligenciada pelos profissionais de saúde. Há uma cultura de que a privação do sono é um símbolo de dedicação à profissão médica, parecendo aumentar a produtividade nos estudos e no atendimento [25]. Entretanto, estudos sugerem que fadiga, sonolência, depressão, esgotamento, insatisfação no trabalho e baixa qualidade de vida podem afetar negativamente o profissionalismo, a qualidade da assistência e a segurança e satisfação do paciente $[24,26]$.

$\mathrm{Na}$ avaliação do WHOQOL-Bref, as facetas que avaliam a saúde do ambiente físico, a oportunidade de lazer e os recursos financeiros para satisfazer as necessidades se apresentaram com um escore "regular". Estudos apontam que as condições insatisfatórias de trabalho, como falta de reconhecimento, remuneração inadequada, ausência de autonomia, instabilidade, carência de infraestrutura e segurança, podem sobrecarregar o residente, gerando estresse e afetando negativamente sua convivência social e qualidade de vida [27].

A maioria dos entrevistados relatou que o programa de residência médica avaliado não fornecia apoio psicopedagógico aos residentes, enquanto parte dos residentes disse não saber se esse apoio existia ou não. Entretanto, uma pequena parcela afirmou existir apoio psicopedagógico na instituição. Essas discrepâncias demonstram o desconhecimento e a ausência da procura de apoio psicopedagógico por parte dos residentes.

Não há na legislação da Residência Médica Brasileira a determinação da obrigatoriedade de um apoio psicopedagógico, contudo, estudos apontam para essa necessidade. O apoio psicológico ao médico residente pode diminuir os níveis de ansiedade, estresse e depressão, e a interferência destes na motivação e no desempenho, sendo fundamental para o melhor aproveitamento da residência médica [10]. Entretanto, mesmo em instituições que oferecem serviço de apoio psicológico aos residentes, a procura pelos profissionais é baixa, conforme evidenciou estudo realizado em uma universidade que oferecia esse serviço. As possíveis causas apontadas foram a dificuldade do residente em se afastar de suas atividades, pois sua ausência teria que ser coberta por algum colega, e o momento em que o profissional se encontra, no qual suas forças e tempo são direcionados ao aprimoramento de sua formação e ao início da vida profissional, relegando a um segundo plano os cuidados com sua vida pessoal [12].

Em relação ao período da residência médica, um estudo identificou qualidade de vida mais prejudicada no primeiro ano de residência do que nos demais, 
apontando como causa o maior nível de estresse nesse período, decorrente da inexperiência em lidar com o estresse ocupacional [5]. Em outro estudo, o maior índice de ansiedade estava presente também durante o primeiro ano de residência, fase de dúvidas e incertezas com relação à especialidade escolhida, com inseguranças para desempenhar a prática médica, além de necessitar de tempo para estudar e realizar pesquisas. Já nos anos seguintes, essas pressões se tornavam menos evidentes [10].

Uma pesquisa com médicos residentes nos Estados Unidos identificou que os sintomas de exaustão emocional foram mais comuns entre os residentes do primeiro ano e diminuíram a cada ano, enquanto que os sintomas de despersonalização que podem se manifestar como cinismo e atitudes cruéis para com os pacientes aumentaram à medida que os anos de residência se passaram. Como possíveis explicações para esses achados foram apontados efeitos acumulados da precoce exaustão emocional da residência, que geram um desgaste do idealismo médico em longo prazo [26].

Ao contrário da literatura citada, na presente pesquisa não foram encontradas diferenças estatisticamente significativas na relação da qualidade de vida com o período de residência. Talvez este achado possa ser explicado pela pequena amostragem, sendo um importante quesito a ser pesquisado em outro momento.

Os médicos residentes entrevistados apresentaram uma qualidade de vida geral e satisfação com a saúde classificadas como "boa". Já a percepção dos sujeitos da própria qualidade de vida apresentou um escore "regular". Essa mesma relação, um escore da autoavaliação inferior ao da qualidade de vida geral, também foi identificada em um estudo com médicos residentes da cidade de Curitiba, PR, em 2010 [6]. Tais dados mostram que o residente percebe sua qualidade de vida mais prejudicada do que ela realmente é.

Em pesquisa com médicos residentes nos Estados Unidos no ano de 2011, os autores perceberam que mesmo com $56,80 \%$ dos residentes classificando sua qualidade de vida como "tão boa quanto pode ser" ou "um pouco boa", 14,80\% classificaram-na como "tão ruim quanto pode ser" ou "um pouco ruim", mostrando que é comum a presença de uma qualidade de vida prejudicada nessa população [26].
Como limitações do presente estudo podemos citar a inclusão de apenas um hospital, o que tornou a amostra pequena e limitada a uma realidade específica, e a ausência de algumas variáveis categorizadas por sexo, que poderiam esclarecer melhor os motivos das diferenças encontradas, o que não invalida a importância dos resultados identificados.

Conclui-se que a análise geral da qualidade de vida apresentou uma média boa, sendo todos os domínios enquadrados nessa classificação, embora algumas facetas tenham recebido o escore "regular". Na autoavaliação, entretanto, a qualidade de vida foi classificada em um escore mais baixo, evidenciando uma percepção regular da própria qualidade de vida. Duas variáveis que apresentaram significância limítrofe em relação à influência negativa na qualidade de vida também merecem destaque: maior carga horária de trabalho com pior satisfação com a saúde e o fato de ter outro emprego com pior percepção da qualidade de vida.

Identificou-se primordialmente o sexo feminino como preditor estatisticamente significativo de pior qualidade de vida geral e também em relação aos domínios físico e psicológico. Com significância estatística limítrofe, constatou-se também que os escores médios nas mulheres da percepção da qualidade de vida e da satisfação com a saúde foram considerados regulares e ficaram abaixo dos referentes aos homens, classificados como bons.

Uma grande importância do presente estudo encontra-se na necessidade de conhecer melhor a realidade dos médicos residentes, em especial das mulheres, na busca constante de promover uma melhor qualidade de ensino e trabalho. São necessárias pesquisas que avaliem mais profundamente a qualidade de vida das médicas residentes, no sentido de verificar os fatores desencadeantes de piores resultados em relação aos homens. Como já citado, a tendência de feminização da medicina está em franco avanço, fato que vem influenciando sobremaneira os modelos de cuidados em saúde e, consequentemente, poderá engendrar novas formas de organização da residência médica. Medidas que visem à melhora da qualidade de vida dos médicos residentes em geral podem se refletir no maior aproveitamento desse período de especialização, resultando em um melhor desempenho profissional.

\section{NOTA}

Declaração de conflitos de interesse

Os autores declaram não haver conflitos de iteresse relevantes ao conteúdo deste estudo. 


\section{REFERÊNCIAS}

1. World Health Organization (WHO). The World Health Organization quality of life assessment (WHOQOL): position paper from the World Health Soc Sci Med. 1995 Nov;41(10):1403-9.

2. Fleck MPA. Problemas conceituais em qualidade de vida. In: A avaliação de qualidade de vida: guia para profissionais de saúde. Porto Alegre: Artmed; 2008. p. 19-28.

3. Rodrigues RTS. Resiliência e características de personalidade de médicos residentes como proteção para o burnout e qualidade de vida [tese]. [São Paulo]: Faculdade de Ciências Médicas da Santa Casa de São Paulo; 2012.

4. Nogueira-Martins LA. Qualidade de vida dos médicos residentes: revisão de estudos brasileiros. Cad ABEM. 2010;6:13-8.

5. Macedo PCM, Cítero VA, Schenkman S, Nogueira-Martins MCF, Morais MB, Nogueira-Martins LA. Health-related quality of life predictors during medical residency in a random, stratified sample of residents. Rev Bras Psiquiatr. 2009;31(2):119-24. http://dx.doi. org/10.1590/S1516-44462009000200007

6. Asaiag PE, Perotta B, Martins MA, Tempski P. Avaliação da qualidade de vida, sonolência diurna e burnout em médicos residentes. Rev Bras Educ Med. 2010;34(3):422-9. http://dx.doi.org/10.1590/S0100-55022010000300012

7. Carvalho CNC, Melo-Filho DA, Carvalho JAG, Amorim ACG. Prevalência e fatores associados aos transtornos mentais comuns em residentes médicos e da área multiprofissional. J Bras Psiquiatr. 2013;62(1):38-45. http://dx.doi.org/10.1590/S0047-20852013000100006

8. Tzischinsky O, Zohar D, Epstein R, Chillag N, Lavie P. Daily and yearly burnout symptoms in Israeli shift work residents. J Hum Ergol (Tokyo). 2001 Dec;30(1-2):357-62.

9. Levey RE. Source of stress for residents and recommendations for programs to assist them. Acad Med. 2001 Feb;76(2):142-50. http:// dx.doi.org/10.1097/00001888-200102000-00010

10. Silva GCC, Sousa EG, Nogueira Martins LA, Buys RC, Santos AASMD, Koch HA. A importância do apoio psicológico ao médico residente e especializando em radiologia e diagnóstico por imagem. Radiol Bras. 2011;44(2):81-4. http://dx.doi.org/10.1590/S010039842011000200006

11. Lourenção LG, Moscardini AC, Soler ZASG. Saúde e qualidade de vida de médicos residentes. Rev Assoc Med Bras. 2010;56(1):81-91. http://dx.doi.org/10.1590/S0104-42302010000100021

12. Souza EM, Gianini RJ, Azevedo-Neto RS, Eluf-Neto J. Perfil do médico residente atendido no Grupo de Assistência Psicológica ao Aluno (Grapal) da FM-USP. Rev Assoc Med Bras. 2009;55(6):684-91. http://dx.doi.org/10.1590/S0104-42302009000600012

13. Aragão J. Introdução aos estudos quantitativos utilizados em pesquisa científicas. Rev Práxis. 2011;3(6):59-62.

14. Fleck MPA, Louzada S, Xavier M, Chachamovich E, Vieira G, Santos L, Pinzon V. Aplicação da versão em português do instrumento abreviado de avaliação da qualidade de vida "WHOQOL-Bref”. Rev Saúde Públ. 2000;34(2):178-83. http://dx.doi.org/10.1590/S003489102000000200012

15. Fleck MPA. O instrumento de avaliação de qualidade de vida da Organização Mundial da Saúde (WHOQOL-100): características e perspectivas. Ciênc Saúde Colet. 2000;5(1):33-8. http://dx.doi.org/10.1590/s1413-81232000000100004

16. Harper A, Power M. Steps for checking and cleaning data and computing domain scores for the WHOQOL-Bref [Internet]. [cited 2016 February 10]. Available from: http://ebooks.pucrs.br/edipucrs/periodicos/ScientiaMedica/diretrizes.pdf

17. Development of the World Health Organization WHOQOL-BREF quality of life assessment. The WHOQOL Group. Psychol Med. 1998 May;28(3):551-8. http://dx.doi.org/10.1017/S0033291798006667

18. Prada MF, Cabrera JG, Torres FG, Ibabe CI, José MP. Calidad de vida relacionada con la salud en una muestra de médicos internos residentes que realizan guardias en un servicio de urgencias: una perspectiva de género. Rev Méd Chile. 2014;142(2):193-8. http://dx.doi org/10.4067/S0034-98872014000200007

19. Scheffer MC, Cassenote AJF. A feminização da medicina no Brasil. Rev Bioét. 2013;21(2): 268-77. http://dx.doi.org/10.1590/S198380422013000200010

20. Fabichak C, Silva-Júnior JS, Morrone LC. Síndrome de burnout em médicos residentes e preditores organizacionais do trabalho. Rev Bras Med Trab. 2014;12(2):79-84.

21. Brasil. Lei n. 6.932 de 07 de julho de 1981. Dispõe sobre as atividades do médico residente e dá outras providências. Presidência da República (1981).

22. Santos MFO, Lucena DP, Oliveira HJ, Bezerra-Júnior J. Avaliação da qualidade de vida dos anestesiologistas da cidade de João Pessoa Rev Ciênc Saúde Nova Esperança. 2011;9(2):33-8.

23. Ribeiro CRF, Silva YMGP, Oliveira SMC. O impacto da qualidade do sono na formação médica. Rev Bras Clin Med. 2014;12(1):8-14.

24. Mordant P, Deneuve S, Rivera C, Carrabin N, Mieog JS, Malyshev N, Van Der Vorst JR, Audisio RA; French Association of Young Surgical Oncologists (AJCO); European Society of Surgical Oncology Young Surgeons and Alumni Club (EYSAC). Quality of life of surgical oncology residents and fellows across Europe. J Surg Educ. 2014 Mar-Apr;71(2):222-8. http://dx.doi.org/10.1016/j.jsurg.2013.07.010

25. Santana, VS, Feitosa AG, Guedes LBA, Sales NBB. Qualidade de vida dos profissionais de saúde em ambiente hospitalar. Rev Pesq Fisiot. 2014;4(1):35-46. http://dx.doi.org/10.17267/2238-2704rpf.v4i1.312

26. West CP, Shanafelt TD, Kolars JC. Quality of life, burnout, educational debt, and medical knowledge among internal medicine residents JAMA. 2011 Sep 7;306(9):952-60. http://dx.doi.org/10.1001/jama.2011.1247

27. Torres AR, Ruiz T, Müller SS, Lima MCP. Qualidade de vida e saúde física e mental de médicos: uma autoavaliação por egressos da Faculdade de Medicina de Botucatu - UNESP. Rev Bras Epidemiol. 2011;14(2):264-75. http://dx.doi.org/10.1590/S1415-790X2011000200008 C 